\section{Therapeutic Potential of Conjugated siRNAs for the Treatment of Major Depressive Disorder}

Major depressive disorder (MDD) is a severe psychiatric syndrome with veryhigh socioeconomic impact worldwide (Global Burden of Disease Study 2013 Collaborators, 2015). This is attributable to three main factors: (i) MDD is a highly prevalent disorder in the general population, (ii) depressive episodes have long duration and occur during active periods of adult life, resulting in very large labor costs, and (iii) standard MDD treatments have limited efficacy, leaving a high percentage of patients with incomplete responses and poor quality of life, thus increasing suicide risk (Rush et al, 2006).

On the basis of clinical and preclinical studies, MDD may be associated with altered structural and synaptic plasticity, stress- or immunerelated pathology, and genetic polymorphisms in brain circuits regulating mood and cognition (Ota and Duman, 2013). In this context, RNA interference (RNAi) may be a useful tool to examine the role of candidate genes in the pathophysiology and treatment of MDD. Hence, RNAi can be used as a therapeutic tool to silence targets involved in the disease process. However, despite its enormous potential, in vivo use of RNAi is limited due to the difficulty to deliver sequences of small RNAs (small interfering RNAsiRNA) to the desired neurons/circuits in mammalian brain. Our strategy has been to develop conjugate siRNA molecules (C-siRNA) in which the siRNA sequence was covalently bound to a selective serotonin reuptake inhibitor (SSRI-sertraline) in order to selectively accumulate it by the dense network of serotonin axon terminals in brain. The amounts (typically 0.5$2 \mathrm{nmol} /$ day) of C-siRNA directed against $5-\mathrm{HT}_{1 \mathrm{~A}}$ receptors $\left(5-\mathrm{HT}_{1 \mathrm{~A}}-\mathrm{R}\right)$ or the serotonin transporter (SERT) were then administered intranasally to mice and C-siRNA sequences were localized into raphe serotonin neurons
(Bortolozzi et al, 2012; Ferrés-Coy et al, 2016). Using this strategy, we discerned the role of pre- and postsynaptic $5-\mathrm{HT}_{1 \mathrm{~A}}-\mathrm{R}$ in response to stress, anxiety phenotype, and response to antidepressant treatments. Interestingly, the selective silencing of presynaptic $5-\mathrm{HT}_{1 \mathrm{~A}}$ autoreceptors was sufficient to elicit antidepressant-like effects in mice thanks to the increased capability of serotonergic neurons to release serotonin during stressful situations (Bortolozzi et al, 2012; FerrésCoy et al, 2013).

Likewise, we employed this approach to silence SERT expression/function. Intranasal administration of a C-siRNA targeting SERT (C-SERT-siRNA) evoked rapid and robust antidepressant-like responses in mice, including elevated forebrain serotonin levels, presynaptic $5-\mathrm{HT}_{1 \mathrm{~A}}-\mathrm{R}$ desensitization, increased hippocampal neurogenesis and expression of trophic factors, and increased dendritic complexity. Further, C-SERT-siRNA reversed depressive-like behaviors in a mouse model of depression. C-SERT-siRNA evoked all these responses in 1 week, whereas SSRI fluoxetine required 1 month (FerrésCoy et al, 2016). In addition, we are using this strategy to knock down other genes potentially involved in stress resilience (eg, TASK-3 channels; Ferrés-Coy et al (2016), unpublished observations) and to target catecholamine neurons, by linking siRNA or antisense oligonucleotide sequences to the respective transporter inhibitors.

Despite the many advantages of siRNA to treat brain diseases, many challenges remain, including off-target effects, rapid degradation, immune response, and poor cellular uptake and selectivity, as well as in vivo delivery. Rational design strategies, predictive models based on secondgeneration algorithms, antibody and chemical modifications, and nanocarriers offer significant opportunities to overcome some of the above problems. Although it is still soon to know the impact of RNAi-based therapies on MDD treatment, our approach to deliver C-siRNA sequences to serotonin neurons through the intranasal route has proven successful in order to elicit rapid and robust antidepressantlike actions in rodents, showing a high potential translational value.

\section{FUNDING AND DISCLOSURE}

This work was supported by grants SAF2015-68346-P (FA) and Retos-Colaboración Subprogram RTC-20142812-1 (AB), Ministry of Economy and Competitiveness (MINECO), and European Regional Development Fund (ERDF), UE; PI13/01390, Instituto de Salud Carlos III, co-financed by ERDF (AB); 20003 NARSAD Independent Investigator $(\mathrm{AB})$; and Centro de Investigación Biomédica en Red de Salud Mental (CIBERSAM). FA and AB are authors of the patent WO/2011/131693 for the siRNA and ASO (antisense oligonucleotides) molecules and the targeting approach related to this work. FA has received consulting honoraria from Lundbeck and he is the Principal Investigator of a grant from Lundbeck. $\mathrm{He}$ is also member of the scientific advisory board of Neurolixis.

\section{ACKNOWLEDGMENTS}

We thank Albert Ferrés-Coy for his outstanding technical assistance.

\section{Francesc Artigas ${ }^{1,2,3}$ and \\ Analia Bortolozzi ${ }^{1,2,3}$}

${ }^{1}$ Department of Neurochemistry and

Neuropharmacology, IIBB-CSIC (Consejo Superior de Investigaciones Científicas), Barcelona, Spain; ${ }^{2}$ Institut d'Investigacions Biomèdiques August Pi i Sunyer (IDIBAPS), Barcelona, Spain; ${ }^{3}$ Centro de Investigación Biomédica en Red de Salud Mental (CIBERSAM),

ISCIII, Madrid, Spain

E-mail: analia.bortolozzi@iibb.csic.es

Bortolozzi A, Castañé A, Semakova J, Santana N Alvarado G, Cortés R et al (2012). Selective siRNAmediated suppression of $5-\mathrm{HT}_{1 \mathrm{~A}}$ autoreceptors evokes strong anti-depressant-like effects. Mol Psychiatry 17: 612-623.

Ferrés-Coy A, Santana N, Castañé A, Cortés R, Carmona MC, Toth M et al (2013). Acute 5- $\mathrm{HT}_{1 \mathrm{~A}}$ autoreceptor knockdown increases antidepressant responses and serotonin release in stressful conditions. Psychopharmacology (Berl) 225: 61-74.

Ferrés-Coy A, Galofré M, Pilar-Cuéllar F, Vidal R, Paz V, Ruiz-Bronchal E et al (2016). Therapeutic antidepressant potential of a conjugated siRNA silencing the serotonin transporter after intranasal administration. $\mathrm{Mol}$ Psychiatry 21: 328-338.

Global Burden of Disease Study 2013 Collaborators (2015). Global, regional, and national incidence, prevalence, and years lived with disability for 301 acute 
and chronic diseases and injuries in 188 countries, 1990-2013: a systematic analysis for the Global Burden of Disease Study 2013. Lancet 386: 743-800.

Ota KT, Duman RS (2013). Environmental and pharmacological modulations of cellular plasticity: role in the pathophysiology and treatment of depression. Neurobiol Dis 57: 28-37.

Rush AJ, Trivedi MH, Wisniewski SR, Nierenberg AA, Stewart JW, Warden D et al (2006). Acute and longer-term outcomes in depressed outpatients requiring one or several treatment steps: a STAR $^{\star}$ D report. Am J Psychiatry 163: 1905-1917.

Neuropsychopharmacology Reviews (2017) 42, 37I-372 doi: 10.1038/npp.2016.182

\section{Error Processing in Depressive States: A Translational Opportunity?}

Cognitive deficits are a core feature of depressive disorders, and have been linked to impaired daily functioning, worse clinical outcome, and treatment resistance. In particular, impairments in cognitive control (i.e., the ability to adaptively modulate action and thoughts based on internal goals and environmental demands) precede depression onset, persist after remission, and predict poor antidepressant responses (Pizzagalli, 2011). Moreover, among healthy participants, better cognitive control (as probed by an emotional Stroop task) predicted lower levels of pro-inflammatory cytokines in response to stress; thus, a better ability to modulate behavior when challenged by emotional information was associated with reduced inflammatory stress reactivity (Shields et al, 2016), highlighting a pathway by which cognitive control and immune function may directly influence one another. These findings suggest that cognitive control deficits might constitute markers of depression vulnerability and thus represent targets for new treatments. As recently emphasized, current pharmacological, psychological, and psychosomatic treatments often fail to ameliorate cognitive deficits despite improving mood (National Academies of Sciences, Engineering, 2015). Accordingly, novel interventions with pro- cognitive effects may offer an innovative strategy to more effectively treat some of the most debilitating-yet often overlooked-symptoms of depression.

Challenges in developing treatments that target cognitive function are compounded by the use of different approaches in preclinical and clinical studies of depression, which unsurprisingly yields poor translation. Preclinical assays frequently involve end points such as time spent struggling in response to stress or latency to eat a novel food, neither of which matches the reality of the human condition or tap into high-level cognitive function. Clinically, cognitive control deficits often emerge when probing error processing. Specifically, individuals with depression show impaired posterror behavioral adjustments (i.e., lower accuracy immediately following an incorrect vs correct response)-a pattern associated with disrupted activation within frontocingulate pathways implicated in cognitive control (Pizzagalli, 2011). Building on these clinical findings, we reported that rats given corticotropin-releasing factor, a peptide that causes myriad signs of stress and depression in humans and laboratory animals, in an attention task showed similar reductions in posterror accuracy. This effect was attenuated by JDTic, a kappa-opioid receptor antagonist with antidepressant-like effects (Beard et al, 2015). These findings indicate that depression-like impairments in cognitive control can be recapitulated in rodents and are sensitive to classes of drugs under investigation for treating depression, opening exciting translational avenues.

Emerging evidence indicates that the same neural markers of cognitive control can be observed in rodents and humans, including potentiated posterror theta oscillations (Laubach et al, 2015) and feedback-related negativity after unsuccessful trials (Warren et al, 2015), which offer additional opportunities to align end points across species. These types of objective neurophysiological markers could be used to screen novel compounds, and prioritize those that normalize behavioral and EEG markers of cognitive control. Creating a framework to study homologous end points in laboratory animals and humans should offer ways to more quickly and accurately predict clinical outcomes in humans, and is consistent with efforts at NIMH (e.g., 'Fast-Fail' trials: http://www.nimh.nih.gov/research-prio rities/research-initiatives/fast-fast-failtrials.shtml) to hasten the development of better therapeutics.

\section{FUNDING AND DISCLOSURE}

Over the past 3 years, DAP has received consulting fees from Akili Interactive Labs, BlackThorn Therapeutics, Otsuka America Pharmaceutical, and Pfizer. WAC has a patent covering the use of kappa-opioid receptor antagonists for the treatment of depression, has received consulting fees from Cerecor, and currently serves as the Editor-inChief of this journal. This study was supported by 1UH2 MH109334 (DAP and WAC), 2R37 MH068376 (DAP), and 2R01 MH063266 (WAC). The author declares no conflict of interest.

\section{Diego A Pizzagalli ${ }^{1}$ and \\ William A Carlezon Jr. ${ }^{1}$}

${ }^{1}$ Department of Psychiatry, Harvard Medical School/ McLean Hospital, Belmont, MA, USA

E-mail: dap@mclean.harvard.edu

Beard C, Donahue RJ, Dillon DG, Van't Veer A, Webber C, Lee J et al (2015). Abnormal error processing in depressive states: a translational examination in humans and rats. Trans/ Psychiatry 12: e564.

Laubach M, Caetano MS, Narayanan NS. Mistakes were made: neural mechanisms for the adaptive control of action initiation by the medial prefrontal cortex. J Physiol Paris 109: 104-117.

National Academies of Sciences, Engineering. Enabling Discovery, Development, And Translation Of Treatments For Cognitive Dysfunction In Depression: Workshop Summary. The National Academies Press: Washington, DC, 2015.

Pizzagalli DA (2011). Frontocingulate dysfunction in depression: toward biomarkers of treatment response. Neuropsychopharmacology 36: 183-206.

Shields GS, Kuchenbecker SY, Pressman SD, Sumida KD, Slavich GM (2016). Better cognitive control of emotional information is associated with reduced pro-inflammatory cytokine reactivity to emotional stress. Stress 19: 63-68.

Warren CM, Hyman JM, Seamans JK, Holroyd CB (2015). Feedback-related negativity observed in rodent anterior cingulate cortex. J Physiol Paris 109: 87-94.

Neuropsychopharmacology Reviews (2017) 42, 372; doi: I 0.1038/npp.2016.183 